\title{
Energy Selective Secondary Electron Detection in SEM for the Characterization of Polymers
}

\author{
C. Rodenburg*, A.J. Pearson**, S.A. Boden***
}

*Department of Materials Science and Engineering, University of Sheffield, Mappin Street, Sheffield, S1 3JD, UK

**Department of Physics and Astronomy, University of Sheffield, Hounsfield Road Sheffield,S3 7RH, UK

***Electronics and Computer Science, University of Southampton, Highfield, Southampton, SO17 1BJ, UK

That differences in detection of SEs can influence the information contained in an SE image has been shown before [1]. Furthermore identification via finger-printing methods based on the SE spectra collected at low acceleration voltage $(1 \mathrm{kV})$ was demonstrated for a number of inorganic materials [2]. So far this method has failed to attract wide spread applications. Ref 2 was focused only on inorganic materials for which X-rays and back scattered electrons may be used for chemical analysis and/ or mapping of different materials, at higher accelerating voltages.

Such analysis is in many cases not expected to be applicable to organic materials. For example polymers are generally composed of light elements such as carbon and hydrogen. If higher atomic number elements are present their concentration is often below the EDX detection limit [3]. In addition high electron doses needed for EDX analysis may cause substantial damage to organic specimens. When SE based analysis at low accelerating voltages is carried out it has the advantage that the number of SE emitted per primary electron, the SE yield, is substantially higher than that at larger primary beam energies. For example a number of polymers were measured to have a maximum yield in the range of $0.2 \mathrm{eV}$ to $0.3 \mathrm{eV}$ with $\mathrm{SE}$ yields $\sim 3$, compared to yields $\sim 0.2$ at $10 \mathrm{keV}$ primary beam energy [4]. This means much smaller primary beam currents can be used at the low beam energies, minimising damage. Although identification by finger printing was only shown for inorganic materials, secondary electron emission of organic materials was also demonstrated to be sensitive to chemistry. For example it was found that SE emission is linked to the number of valence electrons in a monomer and a dependence on the ratio of $\pi$ to $\sigma$ electrons has also been observed [5].

Here we present initial results that demonstrate the usefulness of energy selective SE detection for the analysis of polymers blends used in organic solar cells. P3HT/PCBM (1:0.7) blends were spin cast on silicon substrates followed by annealing for $10 \mathrm{~min}$ in a nitrogen atmosphere. Specimens were imaged in a FEI Sirion S-FEG SEM, where they were imaged in UHR mode using an acceleration voltage of $1 \mathrm{kV}$. Energy selection of the emitted SE is carried using a user adjustable deflection field as described in [1]. In addition a Zeiss Orion Plus TM was used to collect Helium Ion Microscope (HeIM) images.

In Fig 1 the same area of the blend annealed at $170^{\circ} \mathrm{C}$ was imaged using $\mathrm{D}=60 \mathrm{~V}$ (standard, all $\mathrm{SE}$ energies contribute) and $\mathrm{D}=28.4 \mathrm{~V}$ respectively. The contrast was calculated using the standard contrast definition; dividing the intensity difference between particle and matrix at any given location by their respective average intensities. In both cases positive contrast is observed surrounding the particle, as a result of the "edge effect". Particle areas exhibiting this contrast stick 
out as can be seen from the HeIM image in Fig 2. In Fig 1 there is no material contrast between the particle interior and the matrix whereas the energy filtered (lower energy SE only) image exhibits strong material contrast. The lower magnification HeIM image confirms the presence of some material contrast, which is strongly reduced when the magnification is doubled. At a lower annealing temperature of $130^{\circ} \mathrm{C}$ such raised areas were not found but instead relatively flat areas with different composition are present (Fig.3). When imaged in HeIM these areas seem homogeneous, as at high magnifications the material contrast is lost in HeIM (see Fig.3) but energy filtered imaging in at $1 \mathrm{kV}$ in SEM reveals nanoscale compositional variations inside these areas.
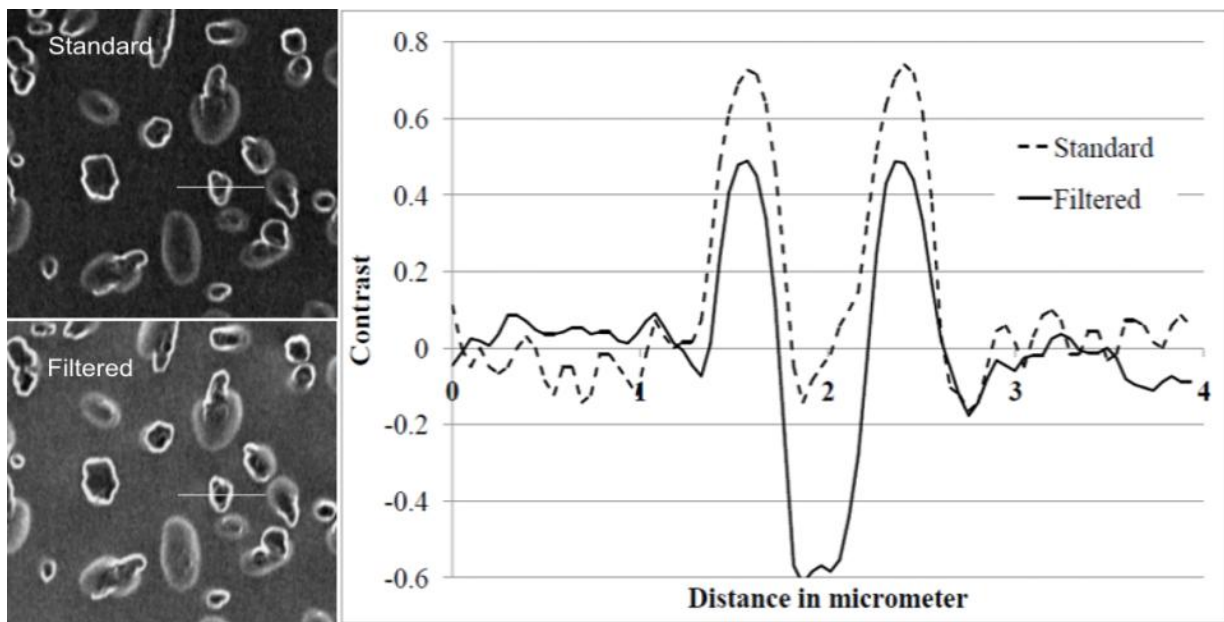

Figure 1 SEM images (standard) and using filtering to select low energy SE only of P3HT:PCBM blend after annealing. Field of view $15 \mu \mathrm{m}$ and contrast profiles obtained from intensity profiles from the locations marked.

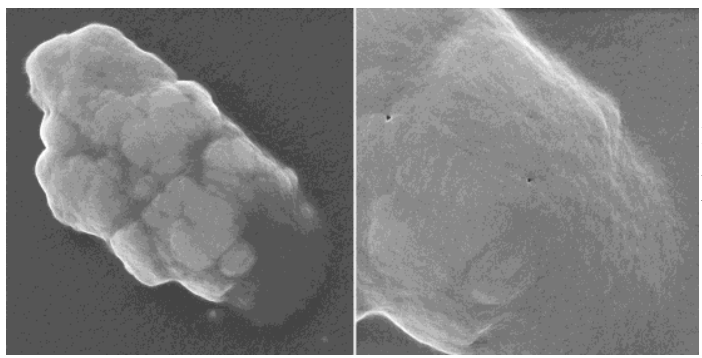

Figure 2: HeIM images of similar particles to that shown in Fig 1. Field of view: left $1 \mu \mathrm{m}$, right: $2 \mu \mathrm{m}$. Note the reduction of contrast at higher magnification, probably due to damage.

Figure 3: Field of view: $300 \mathrm{~nm} \times$ 400nm. Left: Energy filtered SEM image. Note contrast within the particle and high contrast with respect to surrounding blend. Right: HeIM image of same specimen. Particle (marked by white line) similar to that in SEM but showing little contrast to surrounding blend.

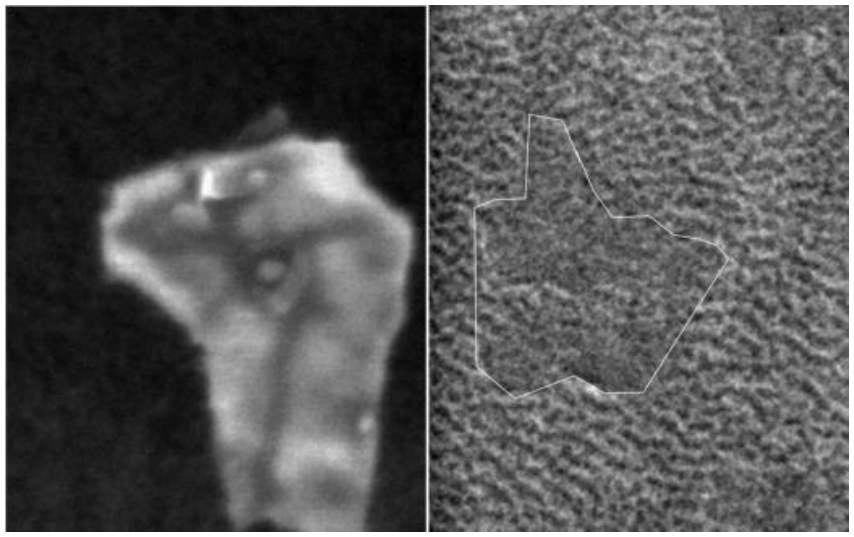

\section{References}

[1] C. Rodenburg et al. Ultramicroscopy 110 (2010) 1185-1191

[2] D.C. Joy et al., Journal of Microscopy, 215 (1) (2004) 77

[3] G.H. Michler, Electron microscopy of polymers, Springer Berlin, London 2008

[4] R.F. Willis, D.K. Skinner, Solid State Communications 13 (1973) 685

[5] Y. Kishimoto et al., Journal of Applied Polymer Science 39 (1990) 2055

[6] C. Rodenburg is supported by the Royal Society. 